\title{
Response inhibition is modulated by functional cerebral asymmetries for facial expression perception
}

\author{
Sebastian Ocklenburg ${ }^{1}{ }^{+}$, Vanessa Ness $^{1+}{ }^{\dagger}$, Onur Güntürkün ${ }^{1}$, Boris Suchan $^{2}$ and Christian Beste ${ }^{3}$ \\ ${ }^{1}$ Biopsychology, Institute of Cognitive Neuroscience, Faculty of Psychology, Ruhr-University Bochum, Bochum, Germany \\ ${ }^{2}$ Neuropsychology, Institute of Cognitive Neuroscience, Faculty of Psychology, Ruhr-University Bochum, Bochum, Germany \\ ${ }^{3}$ Cognitive Neurophysiology, Department of Child and Adolescent Psychiatry, University of Dresden, Dresden, Germany
}

\section{Edited by:}

Hannes Ruge, Technische Universitaet Dresden, Germany

\section{Reviewed by:}

Andres H. Neuhaus, Charité

University Medicine Berlin, Germany

Ulla Martens, Universtity of

Osnabrück, Germany

\section{*Correspondence:}

Sebastian Ocklenburg, Biopsychology, Institute of Cognitive Neuroscience,

Faculty of Psychology, Ruhr-University Bochum, Universitätsstraße 150

44780 Bochum, Germany

e-mail: sebastian.ocklenburg@rub.de

${ }^{\dagger}$ Sebastian Ocklenburg and Vanessa Ness have contributed equally to this work.
The efficacy of executive functions is critically modulated by information processing in earlier cognitive stages. For example, initial processing of verbal stimuli in the languagedominant left-hemisphere leads to more efficient response inhibition than initial processing of verbal stimuli in the non-dominant right hemisphere. However, it is unclear whether this organizational principle is specific for the language system, or a general principle that also applies to other types of lateralized cognition. To answer this question, we investigated the neurophysiological correlates of early attentional processes, facial expression perception and response inhibition during tachistoscopic presentation of facial "Go" and "Nogo" stimuli in the left and the right visual field (RVF). Participants committed fewer false alarms after Nogo-stimulus presentation in the left compared to the RVF. This right-hemispheric asymmetry on the behavioral level was also reflected in the neurophysiological correlates of face perception, specifically in a right-sided asymmetry in the N170 amplitude. Moreover, the right-hemispheric dominance for facial expression processing also affected eventrelated potentials typically related to response inhibition, namely the Nogo-N2 and Nogo-P3. These findings show that an effect of hemispheric asymmetries in early information processing on the efficacy of higher cognitive functions is not limited to left-hemispheric language functions, but can be generalized to predominantly right-hemispheric functions.

\section{Keywords: executive functions, Go/Nogo task, EEG, ERP, laterality, lateralization, Nogo-N2, Nogo-P3}

\section{INTRODUCTION}

Intentional response inhibition is an executive control mechanism that is mainly regulated by the prefrontal cortex (e.g., Chikazoe, 2010). A commonly used method to experimentally assess this cognitive function is the Go/Nogo task in which participants have to perform a simple motor action (e.g., pressing a key on a $\mathrm{PC}$ keyboard) in response to one type of stimulus (Go), while they have to refrain from responding when the other type of stimulus (Nogo) is presented (e.g., Beste et al., 2010, 2013). One important factor modulating performance in Go/Nogo tasks is bottom-up information processing of the used stimuli (Knudsen, 2007), and it has been shown that hemispheric asymmetries for the used stimulus material affect the efficacy of response inhibition. For instance, Ocklenburg et al. (2011) tachistoscopically presented verbal "Go" and "Nogo" stimuli in the left and the right visual field (RVF) and reported that participants committed fewer false alarms when reacting to verbal Nogo-stimuli presented in the RVF than to stimuli presented in the left visual field (LVF), reflecting the well-known left-hemispheric dominance for processing of verbal stimuli (Hugdahl, 2000; Corballis, 2012; Hirnstein et al., 2012; Ocklenburg et al., 2012; Bless et al., 2013; Cai et al., 2013; Ocklenburg et al., 2013). Thus, initial stimulus representation in the non-dominant hemisphere seems to be leading to a less efficient inhibition process, an idea that was also supported by another divided visual field Go/Nogo study with verbal stimuli (Measso and Zaidel, 1990). However, it is unclear whether this effect is specific for the language system or a general principle that also applies to other types of lateralized cognition. Therefore, it was the aim of the present study to investigate whether the efficacy of response inhibition processes is also modulated by a typical rightsided functional asymmetry, the well-known right-hemispheric dominance for face processing (Levine et al., 1988; Rossion et al., 2003; Dien, 2009; Sung et al., 2011; Gainotti, 2013). To this end, we recorded event-related potentials (ERP's) during tachistoscopic presentation of facial "Go" and "Nogo" stimuli in the LVF and RVF.

The earliest ERP component that was assessed was the P1, a positive component with a peak between 80 to $120 \mathrm{~ms}$ after stimulus presentation (Proverbio et al., 2012) which is centered over the occipital cortex (electrodes $\mathrm{O} 1$ and $\mathrm{O} 2$ ). The P1 is the earliest endogenous visual ERP component and is reliably elicited in response to visual stimuli (Taylor, 2002; de Haan et al., 2003). It has been shown to be modulated by a number of factors, including stimulus characteristics and attentional processes (Herrmann and Knight, 2001; Herrmann et al., 2005; Beste et al., 2008; Martin et al., 2008; Wild-Wall et al., 2012). Interestingly, the P1 has been suggested to reflect early face processing (Itier and Taylor, 2002) and it has been found that the P1 is shorter to faces than inverted faces (Taylor, 2002) and that for central stimulus presentation, P1 amplitudes are more positive after presentation of stimuli showing make-up resembling a human face compared to animal-like makeup (Luo et al., 2013). However, there are also studies that did not 
find any effect of faces compared to non-face visual patterns on the P1 (e.g., see Rossion et al., 1999). Findings regarding lateralization of the P1 are ambiguous, with some work reporting no significant side effects (Herrmann et al., 2005) while a recent study by Proverbio et al. (2012) reported that the P1 in a face-sex categorization task was left lateralized in women and bilateral in men.

The second early ERP component that was assessed was the N170 (Bentin etal., 1996; Itier et al., 2006, 2011). The N170 is a negative component which peaks about 130 to $170 \mathrm{~ms}$ after stimulus presentation, is usually centered over the occipito-temporal cortex (Bentin etal., 1996; Eimer, 2000; Rossion and Gauthier, 2002; Rossion et al., 2003; Bieniek et al., 2013). For central stimulus presentation, N170 amplitudes are more negative after presentation of face-like make-up stimuli compared to animal-like make-up stimuli (Luo et al., 2013). Functionally, it is thought to reflect structural encoding of faces (Herrmann et al., 2005). Rossion et al. (2003) reported right lateralization of the N170 for faces. In contrast, it was bilateral for cars and left-lateralized for words. In accordance with these findings, right lateralization of the N170 was also reported by several other studies (e.g., Bentin et al., 1996; Balconi and Lucchiari, 2005; Maurer et al., 2008; Mercure et al., 2008; but see: Proverbio et al., 2010).

In addition to these early ERP components, it is also of interest to assess whether the neurophysiological correlates of response inhibition, such as the Nogo-N2 and Nogo-P3, are modulated by tachistoscopic presentation of facial Go and Nogo stimuli. This is particularly interesting in order to elucidate whether lateralized processing in perceptual and early attentional cognitive processes affect higher cognitive functions such as executive control. The Nogo-N2 is a negative component that is thought to be related to either pre-motor inhibition (Falkenstein et al., 1999) or response conflict (Nieuwenhuis et al., 2003). Ocklenburg etal. (2011) could show that the N2 is lateralized when verbal "Go" and "Nogo" stimuli are presented tachistoscopically in the left and the RVF, so that initial stimulus processing is limited to one hemisphere. In accordance with the conflict hypothesis by Nieuwenhuis et al. (2003), the Nogo-N2 was stronger in response to Nogo-stimuli presented in the LVF. Thus, initial stimulus processing by the subdominant hemisphere leads to a stronger response conflict than initial processing by the dominant hemisphere, even if the inhibition process itself is driven by bilateral prefrontal networks. Apart from the Nogo-N2, the Nogo-P3 has also been related to response inhibition. The Nogo-P3 is a late positive component that has been linked to the evaluation of successful inhibition (Band and van Boxtel, 1999; Roche et al., 2005; Sehlmeyer etal., 2010; Smith etal., 2010, 2013; Beste etal., 2011a). For the Nogo-P3, Ocklenburg etal. (2011) did not observe as clear an asymmetry effect as for the Nogo-N2, but there was a non-significant trend for lateralization on Nogo-trials only.

Based on these findings, we hypothesize that in our task, participants should commit fewer false alarms on Nogo-trials after stimulus presentation in the LVF. This behavioral performance asymmetry should be accompanied by electrophysiological asymmetries on the level of the P1, N170, N2 and possibly P3.

\section{MATERIALS AND METHODS PARTICIPANTS}

Twenty-eight neurologically healthy volunteers (17 female, 11 male) with a mean age of 24.35 years (range: $21-32$ years) participated in the present study. Handedness was assessed using the German version of the Edinburgh Handedness Inventory (EHI; Oldfield, 1971). All participants were right-handed according to the results of EHI (mean laterality quotient 91.5; range 56-100). All participants gave written informed consent and were treated in accordance with the declaration of Helsinki. The study was approved by the ethics committee of the Faculty of Psychology, Ruhr-University Bochum, Germany.

\section{EXPERIMENTAL PARADIGM}

A Go/Nogo task was used to measure response inhibition to face stimuli that were presented tachistoscopically on a $17^{\prime \prime}$ CRT computer monitor. Subjects had to react to "Go"-stimuli by pressing a key on a custom-made reaction-pad, and to refrain from pressing the key after a "Nogo"-stimulus was presented. The stimuli were two morphed male faces taken from the BESST (Bochum Emotional Stimulus Set; Thoma et al., 2012): a friendly and an angry face. To control for possible valence effects of the emotional faces, each participant completed two blocks in randomized order, one block in which the friendly face was the "Go"-stimulus and the angry face was the "Nogo"-stimulus and another block in which the angry face was the "Go"-stimulus and the friendly face was the "Nogo"-stimulus. On half of the trials within each experimental block, subjects responded toward the "GO" stimulus with the index finger of their right hand, and on the other half they responded with their left index finger toward the "GO" stimulus, in randomized order. Overall, the task consisted of 2560 trials (1280 per block), with 1792 trials being "Go"-trials $(70 \%)$ and 768 trials being "Nogo" trials (30\%). On half of the trials, stimuli were presented in the LVF, in the other half in the RVF, in randomized order. At the beginning of the experiment, participants were instructed to place the head on a chin rest placed at a distance of $57 \mathrm{~cm}$ from the monitor. Accordingly, $1 \mathrm{~cm}$ on the screen represented $1^{\circ}$ of visual angle. Stimuli had a maximum width of $3^{\circ}$ visual angle (from ear to ear) and a maximum height of $5^{\circ}$ visual angle (from the neck to the top of the head.). Subjects were instructed to fixate a black fixation cross that was presented in the middle of the screen throughout the experiment. Each trial started with tachistoscopic presentation of the stimulus for $185 \mathrm{~ms}$. Afterward, the central fixation cross was presented for $365 \mathrm{~ms}$ (Ocklenburg et al., 2011). The inter-trial interval was randomized between 750 and $950 \mathrm{~ms}$. Only the central fixation cross was presented during this interval.

\section{EEG RECORDING AND ANALYSIS}

EEG data were recorded from 65 active $\mathrm{Ag}-\mathrm{AgCl}$ electrodes at standard scalp positions against a reference electrode located at FCz. Data were recorded with a sampling rate of $1000 \mathrm{~Hz}$, and down-sampled off-line to $128 \mathrm{~Hz}$. All electrode impedances were kept below $5 \mathrm{k} \Omega$. The data was band-pass filtered (0.5$20 \mathrm{~Hz}$ ) offline before further data processing and then visually inspected to remove technical artifacts. Horizontal and vertical 
eye movements as well as pulse artifacts were then corrected using an independent component analysis (ICA; Infomax algorithm) applied to the un-epoched data set. In the epoched data, automated artifact rejection procedures with the following rejection criteria were applied: maximum voltage steps of more than $50 \mu \mathrm{V} / \mathrm{ms}$, maximal value differences of $200 \mu \mathrm{V}$ in a $200 \mathrm{~ms}$ interval, or activity below $0.1 \mu \mathrm{V}$. To achieve a reference-free evaluation, peak, and latency analyses were performed after calculation of current source density (CSD) of the signals (Perrin et al., 1989). For statistical analysis, amplitudes were quantified relative to a baseline covering $200 \mathrm{~ms}$ before stimulus presentation until stimulus onset. Averaging was locked at the time point of "Go"- or "Nogo"-stimulus presentation and analysis epochs had a length of $1500 \mathrm{~ms}$ (from $200 \mathrm{~ms}$ before stimulus presentation until $1300 \mathrm{~ms}$ after stimulus presentation). Subsequent to averaging, P1, N170, and N2 amplitudes in "Go"- and "Nogo"-trials were calculated relative to baseline using only trials on which participants had reacted correctly. P3 amplitudes were calculated relative to N2 amplitudes. For each ERP component, the local maximum (for positive components) or minimum (for negative components) within a given time window (P1: 50$150 \mathrm{~ms}$ after stimulus presentation; N170: 100-200 ms; N2: 200-400 ms; P3: 250-550 ms) was determined. This was done using a semi-automated search function implemented in the analysis software. The results of the automated search were then visually inspected and corrected of necessary. For the P1, amplitudes and latencies were quantified at the standard positions $\mathrm{O} 1$ and O2, while for the N170, amplitudes and latencies were quantified at electrodes CP5 and CP6. For the Nogo-N2 and Nogo-P3, amplitudes and latencies were quantified at the standard position FCz.

\section{STATISTICAL ANALYSIS}

The behavioral data (i.e., rate of false alarms on Nogo trials as well as misses and reaction times on Go-trials) were analyzed using paired samples t-tests to compare performance after stimulus presentation in the LVF and RVF. P1 and N170 data were analyzed using repeated measures analyses of variance (ANOVAs) with the within-subjects factors electrode (P1: O1 and O2; N170: CP5 and CP6), condition (Go, Nogo), and visual half-field (RVF, LVF). $\mathrm{N} 2$ and $\mathrm{P} 3$ data were analyzed using repeated measures ANOVAs with the within-subjects factors condition (Go, Nogo) and visual half-field (RVF, LVF). When appropriate, the degrees of freedom were adjusted using Greenhouse-Geisser correction. The $p$-levels for post hoc testing were adjusted using Bonferroni correction. Effect sizes are provided as the proportion of variance accounted for (partial $\eta^{2}$ ). As a measure of variability, the standard error of the mean (SEM) was used. All statistical analyses were conducted using IBM SPSS Statistics 20.

\section{RESULTS}

\section{BEHAVIORAL DATA}

In Nogo-trials, the false alarm rate was higher for stimuli that were presented in the RVF $(29.82 \% \pm 3.77)$ than for stimuli that were presented in the $\operatorname{LVF}\left(25.69 \% \pm 3.12 ; t_{(27)}=2.39 ; p<0.05\right)$. In contrast, no visual field difference was observed for the number of misses on Go-trials (RVF: $8.43 \% \pm 2.16$; LVF: $8.43 \% \pm 2.29$; $\left.t_{(27)}=0.01 ; p=0.99\right)$ or reaction time on correct Go-trials (RVF: $472.09 \mathrm{~ms} \pm 11.19$; LVF: $468.11 \mathrm{~ms} \pm 11.22 ; t_{(27)}=-1.14$; $p=0.27)$.

\section{NEUROPHYSIOLOGICAL DATA}

P1

For P1 amplitudes (see Figure 1), the ANOVA revealed a main effect of electrode $\left[F_{(1,27)}=4.42 ; p<0.05\right.$; partial $\left.\eta^{2}=0.14\right]$, indicating a more positive amplitude of the $\mathrm{P} 1$ at the left-sided electrode $\mathrm{O} 1(24.47 \pm 2.94)$ compared to the right-sided electrode $\mathrm{O} 2(18.86 \pm 3.07)$. In addition, a significant interaction visual half-field $\times$ electrode $\left[F_{(1,27)}=4.38 ; p<0.05\right.$; partial $\left.\eta^{2}=0.14\right]$ indicated that after stimulus presentation in the RVF, the P1 was more positive at the left-sided electrode $\mathrm{O} 1(27.71 \pm 4.08)$ than at the right-sided electrode $\mathrm{O} 2(16.01 \pm 2.59$; Bonferroni corrected
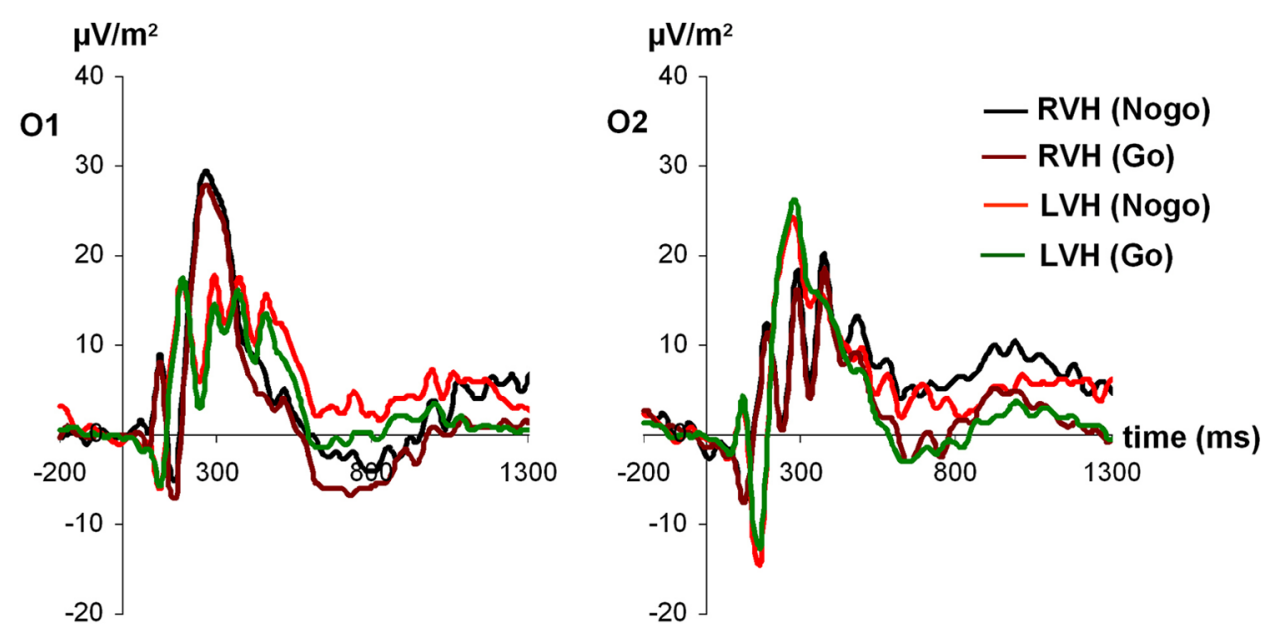

FIGURE 1 | Time course of ERP components at electrodes 01 and 02 in the Go and Nogo condition after stimulus presentation in the left and right visual field. Time point 0 indicates the point of Go- or Nogo-stimulus presentation. 
post hoc test: $p<0.01)$. In contrast, after stimulus presentation in the LVF, no amplitude difference between the two electrodes was observed (O1: $21.22 \pm 2.62 ; \mathrm{O} 2: 21.71 \pm 4.12$; Bonferroni corrected post hoc test: $p=1.00)$. Moreover, a significant interaction visual half-field $\times$ condition emerged $\left[F_{(1,27)}=4.35\right.$; $p<0.05$; partial $\left.\eta^{2}=0.14\right]$, indicating a visual half-field difference between Go- and Nogo-trials, but both post hoc tests failed to reach significance, indicating a rather weak effect (Gotrials: LVF: $21.40 \pm 2.67$; RVF: $20.40 \pm 3.05$; Bonferroni corrected post hoc test: $p=1.00$; Nogo-trials: LVF: $21.53 \pm 2.86$; RVF: $23.32 \pm 2.88$; Bonferroni corrected post hoc test: $p=0.74$ ). All other main effects and interactions failed to reach significance (all $p>0.11)$.

For P1 latencies, only the visual half-field $\times$ condition interaction reached significance $\left[F_{(1,27)}=4.37 ; p<0.05\right.$; partial $\left.\eta^{2}=0.14\right]$, indicating a trend toward a smaller P1 latency on Nogo-trials after stimulus presentation in the LVF (126.27 $\mathrm{ms} \pm 9.20$ ) compared to the RVF (RVF: $139.23 \mathrm{~ms} \pm 8.79$; Bonferroni corrected post hoc test: $p=0.33$; Go-trials: LVF: $140.49 \mathrm{~ms} \pm 9.96$; RVF: $132.95 \mathrm{~ms} \pm 10.30$; Bonferroni corrected post hoc test: $p=0.80$ ). However, since both post hoc tests failed to reach significance, this effect seems to be rather weak. Moreover, a trend toward a visual half-field $\times$ electrode interaction emerged $\left[F_{(1,27)}=3.46 ; p=0.07\right.$; partial $\left.\eta^{2}=0.11\right]$. All other main effects and interactions failed to reach significance (all $p>0.11$ ).

\section{N170}

For N170 amplitudes (see Figure 2), the ANOVA revealed a significant main effect of condition $\left[F_{(1,27)}=4.48\right.$; $p<0.05$; partial $\left.\eta^{2}=0.14\right]$, indicating that the N170 was more negative on Nogotrials $(-15.76 \pm 1.20)$ compared to Go-trials $(-14.34 \pm 1.12)$. Moreover, an interaction visual half-field $\times$ electrode emerged $\left[F_{(1,27)}=17.08 ; p<0.001 ;\right.$ partial $\left.\eta^{2}=0.39\right]$, indicating that after presentation of a face in the LVF, the N170 was more negative at the right-sided electrode CP6 $[-19.03 \pm 1.87]$ than at the leftsided electrode CP5 $(-12.68 \pm 1.56$, Bonferroni-corrected post hoc test: $p<0.05]$. For presentation of a face in the RVF, a trend toward the opposite direction was observed (CP5: $-16.05 \pm 1.71$;
CP6: $-12.45 \pm 1.35)$, but the post hoc test failed to reach significance $(p=0.19)$. In addition, a trend toward a condition $\times$ visual half-field $\times$ electrode emerged $\left[F_{(1,27)}=2.88 ; p=0.10\right.$; partial $\left.\eta^{2}=0.10\right]$. All other main effects and interactions failed to reach significance (all $p>0.13$ ). The visual half-field $\times$ electrode interaction also reached significance for $\mathrm{N} 170$ latency $\left[F_{(1,27)}=6.25\right.$; $p<0.05$; partial $\eta^{2}=0.19$ ]. After presentation of a face in the LVF, the N170 had a longer latency at the right-sided electrode CP6 (173.55 ms \pm 5.14$)$ than at the left-sided electrode CP5 (147.18 ms \pm 9.48 , Bonferroni-corrected post hoc test: $p<0.05$ ). For presentation of a face in the RVF, no significant difference between electrodes was observed (CP5: $173.97 \mathrm{~ms} \pm 9.13$; CP6: $165.46 \mathrm{~ms} \pm 8.08$; Bonferroni-corrected post hoc test: $p=0.80$ ). All other main effects and interactions failed to reach significance (all $p>0.11$ ).

\section{N2 and $P 3$}

For N2 amplitudes (see Figure 3), the ANOVA revealed a significant main effect of condition $\left[F_{(1,27)}=6.45 ; p<0.05\right.$; partial $\left.\eta^{2}=0.19\right)$, indicating that the $\mathrm{N} 2$ was more negative on Nogo-trials $(-14.06 \pm 1.71)$ than on Go-trials $(-10.57 \pm 1.36)$. Moreover, a significant main effect of visual half-field emerged $\left[F_{(1,27)}=4.91 ; p<0.05\right.$; partial $\left.\eta^{2}=0.15\right]$, indicating that the $\mathrm{N} 2$ was more negative after stimulus presentation in the RVF $(-14.25 \pm 1.91)$ than after stimulus presentation in the LVF $(-10.37 \pm 1.32)$. The visual half-field $\times$ condition interaction failed to reach significance $(p=0.36)$. For N2 latencies, all effects failed to reach significance (all $p>0.11$ ).

Due to N2 amplitude differences, P3 amplitudes were not determined peak-to-baseline but peak-to-peak, with the N2 serving as baseline. Only the main effect of condition reached significance $\left[F_{(1,27)}=13.99 ; p<0.05\right.$; partial $\left.\eta^{2}=0.34\right]$, indicating that $\Delta$ was larger on Nogo-trials $(23.50 \pm 2.43)$ than on Go-trials $(15.21 \pm 1.96)$. All other effects failed to reach significance (all $p>0.20)$.

For P3 latencies, the main effect of visual half-field reached significance $\left[F_{(1,27)}=6.07 ; p<0.05 ;\right.$ partial $\left.\eta^{2}=0.18\right]$, indicating that the P3 had a longer latency after stimulus presentation in
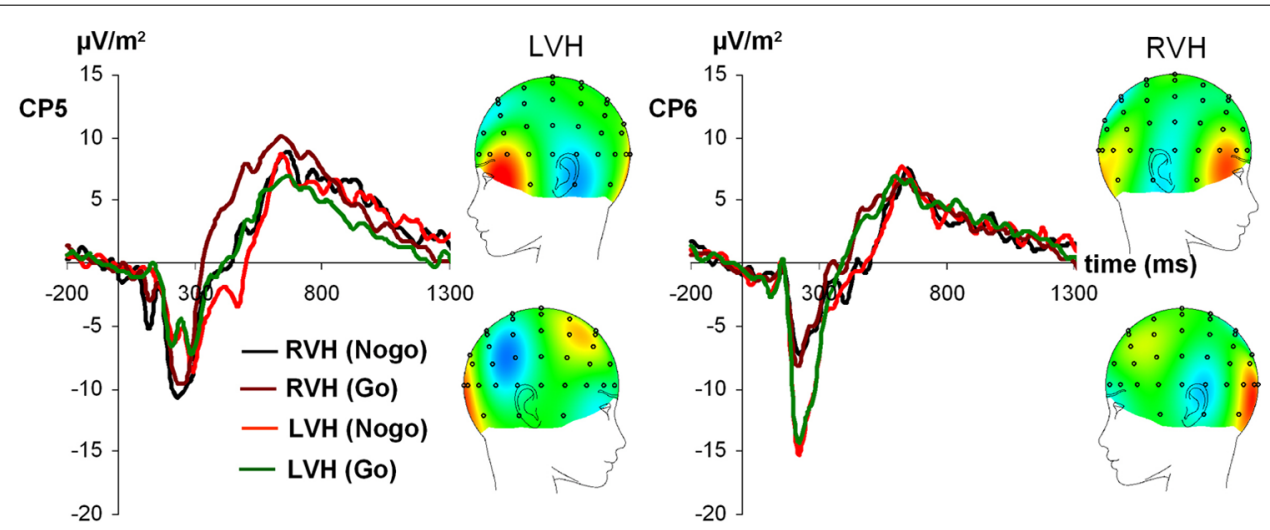

FIGURE 2 |Time course of ERP components at electrodes CP5 and CP6 in the Go and Nogo condition after stimulus presentation in the left and right visual field. Time point 0 indicates the point of Go- or Nogo-stimulus presentation. 


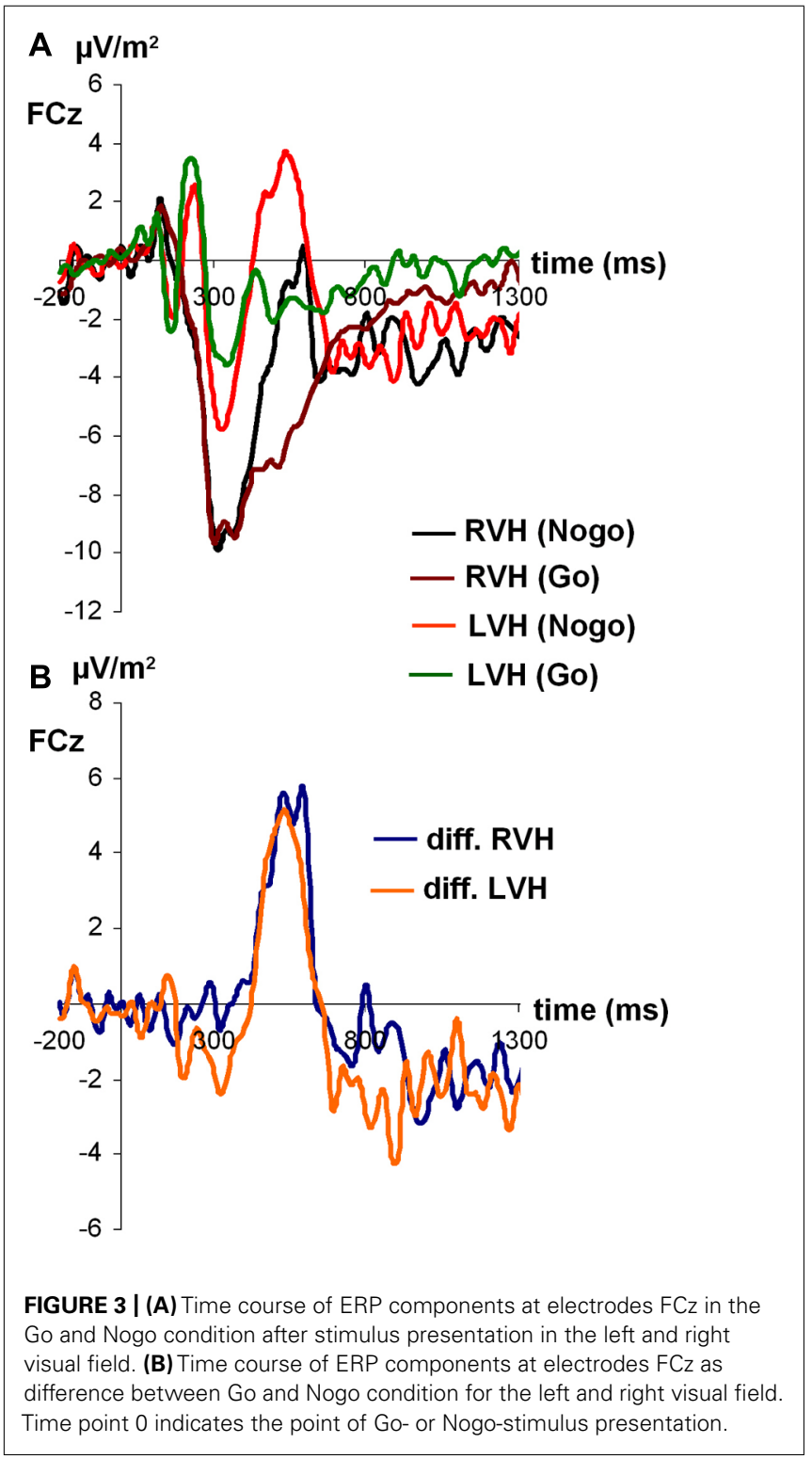

the RVF (492.89 $\mathrm{ms} \pm 20.11)$ than after stimulus presentation in the LVF ( $410.30 \mathrm{~ms} \pm 27.31$ ). This effect was modulated by condition, as indicated by a significant interaction visual halffield $\times$ condition $\left[F_{(1,27)}=5.99 ; p<0.05\right.$; partial $\left.\eta^{2}=0.18\right]$. Interestingly, the visual half-field difference reached significance only on Nogo-trials (LVF: $399.28 \pm 31.14$; RVF: $551.89 \pm 26.38$; Bonferroni-corrected post hoc test: $p<0.01$ ), but not on Go-trials (LVF: $421.32 \pm 38.99$; RVF: $433.87 \pm 33.59$; Bonferroni-corrected post hoc test: $p=1.00)$. The main effect of condition failed to reach significance $(p=0.18)$.

Since there is some controversy surrounding the use of the peak amplitude as a measure for the P3 (Luck, 2005), we also calculated the mean amplitude from 400 to $500 \mathrm{~ms}$ after stimulus presentation as an alternative measure for the $\mathrm{P} 3$. As for the peak amplitude, the main effect of condition reached significance $\left[F_{(1,27)}=10.95\right.$; $p<0.01$; partial $\eta^{2}=0.29$ ], indicating the $\mathrm{P} 3$ was more positive on Nogo-trials $(1.21 \pm 2.32)$ than on Go-trials $(-4.18 \pm 1.51)$. Moreover, the main effect of visual half-field reached significance $\left[F_{(1,27)}=6.90 ; p<0.05\right.$; partial $\left.\eta^{2}=0.20\right]$, indicating a more positive P3 after stimulus presentation in the $\operatorname{LVF}(1.71 \pm 1.96)$ than after stimulus presentation in the RVH $(-4,68 \pm 2.33)$. The interaction failed to reach significance $(p=0.09)$.

\section{DISCUSSION}

Functional cerebral asymmetries have been shown to modulate the efficacy of executive functions (Measso and Zaidel, 1990; Ocklenburg et al., 2013). While previous studies investigated how the left-hemispheric language dominance affects these prefrontally mediated cognitive functions, the present study was aimed at answering the question how executive functions are modulated by the right-hemispheric dominance for face processing. To this end, we recorded ERPs on a tachistoscopic version of the classic Go/Nogo task in which faces were presented in the left and RVF.

Behaviorally, participants committed fewer false alarms on Nogo-trials after stimulus presentation in the LVF. In line with the results of several earlier studies using the divided visual field technique with face stimuli (Leehey and Cahn, 1979; Young and Bion, 1981; Levine and Koch-Weser, 1982; Young, 1984; Young et al., 1985; Gainotti, 2013), this finding indicates greater efficacy of the right hemisphere for facial expression perception. In contrast, no hemispheric asymmetries were observed for accuracy or reaction times on Go-trials, which may be attributed to low task demands in the Go-condition possibly resulting in a ceiling effect. Moreover, this finding is also in line with the behavioral results of earlier studies that used divided visual fields versions of the Go/Nogo Task with verbal stimuli. These studies found that response inhibition is more efficient when initial stimulus processing is performed by the dominant hemisphere (Measso and Zaidel, 1990; Ocklenburg et al., 2013). Our findings indicate that this connection between functional hemispheric asymmetries and executive functions is not limited to left-hemispheric language function, but can also be observed for right-hemispheric functions.

In the ERP data, asymmetries were observed for various components in different cognitive processing stages. In accordance with the results of Proverbio et al. (2012) for female participants, we found left lateralization of the $\mathrm{P} 1$ after stimulus presentation in the RVF. Stimulus presentation in the LVF, however, did not lead to any asymmetry effects. This finding further supports the assumption of Proverbio et al. (2012) that for some types of face-processing tasks at least some left-hemispheric functions are necessary. Specifically, Proverbio et al. (2012) argued that facial tasks which require a high amount of local feature analyses may lead to left-lateralization of the P1 because local visual analyses are known to activate more left-hemispheric networks than global visual analyses (e.g., Hellige, 1996; Yovel et al., 2001). Since we used emotional faces in the present study, which differed mainly with regard to those parts of the face that communicate emotions (e.g., mouth, eyes, and eye-brows), one could speculate that participants partly relied on local visual analysis of these face features to react correctly, ultimately leading to the observed left-lateralization of the P1.

For the N170, the largest negative amplitude was observed at the right-sided electrode CP6 after stimulus presentation in the LVF. 
Moreover, only after stimulus presentation in the LVF (and thus initial stimulus processing in the dominant right hemisphere), did a significant amplitude difference between right- and left-sided electrodes emerge. Here, the N170 had a more negative amplitude at the right compared to the left electrode site. After stimulus presentation in the RVF (and thus initial stimulus processing in the non-dominant left hemisphere), no electrode difference was observed. Thus, in line with other studies reporting right-sided lateralization of the N170 (e.g., Bentin et al., 1996; Rossion et al., 2003; Balconi and Lucchiari, 2005; Maurer et al., 2008; Mercure et al., 2008), our results further support the assumption that the N170 is specifically driven by right-hemispheric brain areas, e.g., the fusiform gyrus or the superior temporal sulcus (Schweinberger et al., 2002; Shibata et al., 2002; Dalrymple et al., 2011). In contrast to the clear right-lateralization of the N170 amplitudes, the N170 had a longer latency over the right than over the left hemisphere when a stimulus was presented in the LVF. Interestingly, a similar finding has also been reported by Proverbio et al. (2012) for central stimulus presentation. In line with the discussion of the P1 findings, this result could be indicative of a need for lefthemispheric processing for certain aspects of our task, e.g., a local feature analysis of the emotional content of the face.

In general, the Nogo-N2 and Nogo-P3 data indicated that our tachistoscopic divided visual field version of the Go/Nogo task worked as intended, since we observed the typical pattern of results for these components. In accordance with previous studies utilizing this paradigm with central stimulus presentation (Beste et al., 2011b; Smith and Douglas, 2011), the Nogo-N2 was more negative after Nogo- than after Go-stimuli, and the Nogo-P3 was more positive after Nogo- than after Go-stimuli. For central stimulus presentation, both the Nogo-N2 and the Nogo-P3 are focused over fronto-central electrode sites (Falkenstein, 2006; Beste et al., 2010) and their generators have been localized mainly in the orbitofrontal cortex (Beste et al., 2010), with some authors reporting a right-shifted topography (Falkenstein, 2006), the inferior frontal cortex (Aron et al., 2004), and the anterior cingulate cortex (Nieuwenhuis et al., 2003).

In contrast to Ocklenburg et al. (2011) who found that the Nogo-N2 was stronger in response to Nogo-stimuli initially processed by the subdominant hemisphere, we found that for facial stimuli, the Nogo-N2 was more negative after initial processing in the subdominant hemisphere, regardless of condition. This difference between the two studies could possible indicate a response conflict in the Go-condition in the present study. For example, the higher stimulus complexity in the present paradigm could have rendered it more difficult for participants to react correctly on both Go and Nogo-trials than in the study by Ocklenburg et al. (2011). This assumption is supported by false alarm rates being overall higher in the present study than in studies using verbal stimuli (present study: RVF: 29.82\%; LVF: 25.69\%; Measso and Zaidel, 1990: RVF: 11.8\%; LVF: 20.1\%; Ocklenburg et al., 2011: RVF: $12.9 \%$; LVF: 16.4\%). Moreover, the miss rate for go-stimuli was around $8 \%$ in the present study, indicating that even when being asked to execute the predominant go-reaction, participants sometimes experienced problems to perform correctly. In addition to the generally higher complexity of the facial stimuli used in the present study, verbal stimuli typically used in Go/Nogo tasks (e.g., the words "Press" and "Stop") are usually highly overlearned, since they have been associated with performing a reaction or refraining from doing so in everyday life. In contrast, in the present study, participants had to learn which stimuli represented a Go-signal or Nogo-signal during the experiment.

In addition to the Nogo-N2 results, we also observed an effect of functional cerebral asymmetries for facial expression perception on Nogo-P3 latencies. On Nogo-, but not on Go-trials, the P3 had a longer latency if initial stimulus processing was conducted by the non-dominant left hemisphere. Thus, initial stimulus processing by the dominant right hemisphere leads to faster evaluation of the inhibition process (Band and van Boxtel, 1999; Roche et al., 2005; Smith et al., 2010, 2013).

There are a few methodological considerations that have to be taken into account when interpreting the present ERP results. First of all, the P1 effects seem to be rather weak, since the half-field $\times$ condition interaction reached significance for both amplitudes and latencies, but both post hoc tests failed to reach significance for both variables after Bonferroni correction. This potential issue might be due to the fact that the $\mathrm{P} 1$ is not specifically elicited by perception of faces, but by perception of visual stimuli in general (Taylor, 2002; de Haan et al., 2003). To address this potential lack of statistical power to reliably detect P1 asymmetry effects, future studies investigating this topic should test larger samples and use a higher number of trials than the present work. One methodological consideration that has to be taken into account when interpreting the $\mathrm{N} 2$ and $\mathrm{P} 3$ results is the fact that it is not clear to what extent results obtained in a paradigm with lateralized stimulus presentation allow to draw conclusions about the impact of hemispheric asymmetries when stimuli are presented in the center of the visual field. For example, ERP studies in the field of hemispheric asymmetries in global vs. local processing demonstrate that central vs. lateralized presentation could affect the occurrence of hemispheric asymmetries: while all ERP studies with central stimulus presentation reported hemispheric asymmetries, some studies with laterally presented stimuli failed to replicate this finding (see Volberg and Hübner, 2004, for an overview). Thus, it would be interesting for futures studies investigating the impact of lateralization on executive functions to include a condition with central stimulus presentation in addition to stimulus presentation in the LVH and RVF. In regard to the present results, this would allow to differentiate hemispheric asymmetries for centrally presented faces (e.g., as reported by Rossion et al., 2003, for the N170) from hemispheric asymmetries following laterally presented stimuli.

Taken together, the present findings show that hemispheric asymmetries in information processing as reflected by early ERP components such as the N170 affect behavioral performance indicators as well as neurophysiological correlates of higher cognitive functions. In principle, initial stimulus processing by the dominant hemisphere leads to more efficient execution of subsequent cognitive tasks, even if task-related ERP components are mediated by bilateral neuronal networks, as is the case for Nogo-N2 and Nogo-P3 (Ocklenburg et al., 2011). This principle is not limited to left-hemispheric language functions, as has been suggested by previous studies, but can also be applied to predominantly right-hemispheric functions. However, it is obvious that the results 
for facial stimuli do not completely mirror the results for verbal stimuli. Thus, the present findings also indicate that it is important to consider both the specific neurobiological properties of the involved cognitive system as well as stimulus variables such as complexity when investigating the impact of functional cerebral asymmetries in information processing on higher cognitive systems.

\section{ACKNOWLEDGMENTS}

This research was supported by a DFG grant to Christian Beste (BE4045/10-1). The funders had no role in study design, data collection and analysis, decision to publish, or preparation of the manuscript.

\section{REFERENCES}

Aron, A. R., Robbins, T. W., and Poldrack, R. A. (2004). Inhibition and the right inferior frontal cortex. Trends Cogn. Sci. 8, 170-177. doi: 10.1016/j.tics.2004. 02.010

Balconi, M., and Lucchiari, C. (2005). Event-related potentials related to normal and morphed emotional faces. J. Psychol. 139, 176-192. doi: 10.3200/JRLP.139.2.176192

Band, G. P., and van Boxtel, G. J. (1999). Inhibitory motor control in stop paradigms: review and reinterpretation of neural mechanisms. Acta Psychol. 101, 179-211. doi: 10.1016/S0001-6918(99)00005-0

Bentin, S., Allison, T., Puce, A., Perez, E., and McCarthy, G. (1996). Electrophysiological studies of face perception in humans. J. Cogn. Neurosci. 8, 551-565. doi: 10.1162/jocn.1996.8.6.551

Beste, C., Baune, B. T., Domschke, K., Falkenstein, M., and Konrad, C. (2010). Dissociable influences of NR2B-receptor related neural transmission on functions of distinct basal ganglia circuits. Neuroimage 52, 309-315. doi: 10.1016/j.neuroimage.2010.04.022

Beste, C., Domschke, K., Radenz, B., Falkenstein, M., and Konrad, C. (2011a). The functional 5-HT1A receptor polymorphism affects response inhibition processes in a context-dependent manner. Neuropsychologia 49, 2664-2672. doi: 10.1016/j.neuropsychologia.2011.05.014

Beste, C., Ness, V., Falkenstein, M., and Saft, C. (2011b). On the role of fronto-striatal neural synchronization processes for response inhibition evidence from ERP phase-synchronization analyses in pre-manifest Huntington's disease gene mutation carriers. Neuropsychologia 49, 3484-3493 doi: 10.1016/j.neuropsychologia.2011.08.024

Beste, C., Konrad, C., Uhlmann, C., Arolt, V., Zwanzger, P., and Domschke, K. (2013). Neuropeptide S receptor (NPSR1) gene variation modulates response inhibition and error monitoring. Neuroimage 71, 1-9. doi: 10.1016/j.neuroimage.2013.01.004

Beste, C., Saft, C., Andrich, J., Gold, R., and Falkenstein, M. (2008). Stimulusresponse compatibility in Huntington's disease: a cognitive-neurophysiological analysis. Neurophysiology 99, 1213-1223. doi: 10.1152/jn.01152.2007

Bieniek, M. M., Frei, L. S., and Rousselet, G. A. (2013). Early ERPs to faces: aging, luminance, and individual differences. Front. Psychol. 4:268. doi: 10.3389/fpsyg. 2013.00268

Bless, J. J., Westerhausen, R., Arciuli, J., Kompus, K., Gudmundsen, M., and Hugdahl, K. (2013). "Right on all occasions?" - on the feasibility of laterality research using a smartphone dichotic listening application. Front. Psychol. 4:42. doi: 10.3389/fpsyg.2013.00042

Cai, Q., Van der Haegen, L., and Brysbaert, M. (2013). Complementary hemispheric specialization for language production and visuospatial attention. Proc. Natl. Acad. Sci. U.S.A. 110, E322-E330. doi: 10.1073/pnas.1212956110

Chikazoe, J. (2010). Localizing performance of go/no-go tasks to prefrontal cortical subregions. Curr. Opin. Psychiatry 23, 267-272. doi: 10.1097/YCO.0b013e3283387a9f

Corballis, M. C. (2012). Lateralization of the human brain. Prog. Brain Res. 195, 103-121. doi: 10.1016/B978-0-444-53860-4.00006-4

Dalrymple, K. A., Oruç, I., Duchaine, B., Pancaroglu, R., Fox, C. J., Iaria, G., et al. (2011). The anatomic basis of the right face-selective N170 in acquired prosopagnosia: a combined ERP/fMRI study. Neuropsychologia 49, 2553-2563. doi: 10.1016/j.neuropsychologia.2011.05.003 de Haan, M., Johnson, M. H., and Halit, H. (2003). Development of face-sensitive event-related potentials during infancy: a review. Int. J. Psychophysiol. 51, 45-58. doi: 10.1016/S0167-8760(03)00152-1

Dien, J. (2009). A tale of two recognition systems: implications of the fusiform face area and the visual word form area for lateralized object recognition models. Neuropsychologia 47, 1-16. doi: 10.1016/j.neuropsychologia.2008.08.024

Eimer, M. (2000). Effects of face inversion on the structural encoding and recognition of faces. Evidence from event-related brain potentials. Brain Res. Cogn. Brain Res. 10, 145-158. doi: 10.1016/S0926-6410(00)00038-0

Falkenstein, M. (2006). Inhibition, conflict and the Nogo-N2. Clin. Neurophysiol. 117, 1638-1640. doi: 10.1016/j.clinph.2006.05.002

Falkenstein, M., Hoormann, J., and Hohnsbein, J. (1999). ERP components in Go/Nogo tasks and their relation to inhibition. Acta Psychol. 101, 267-291. doi: 10.1016/S0001-6918(99)00008-6

Gainotti G. (2013). Laterality effects in normal subjects' recognition of familiar faces, voices and names. Perceptual and representational components. Neuropsychologia 51, 1151-1160. doi: 10.1016/j.neuropsychologia.2013.03.009

Hellige, J. B. (1996). Hemispheric asymmetry for visual information processing Acta Neurobiol. Exp. 56, 485-497.

Herrmann, M. J., Ehlis, A. C., Ellgring, H., and Fallgatter, A. J. (2005). Early stages (P100) of face perception in humans as measured with event-related potentials (ERPs). J. Neural. Transm. 112, 1073-1081. doi: 10.1007/s00702-004-0250-8

Herrmann, C. S., and Knight, R. T. (2001). Mechanisms of human attention: event related potentials and oscillations. Neurosci. Biobehav. Rev. 25, 465-476. doi: 10.1016/S0149-7634(01)00027-6

Hirnstein, M., Westerhausen, R., Korsnes, M. S., and Hugdahl, K. (2012). Sex differences in language asymmetry are age-dependent and small: a large-scale, consonant-vowel dichotic listening study with behavioral and fMRI data. Cortex 49, 1910-1921. doi: 10.1016/j.cortex.2012.08.002

Hugdahl, K. (2000). Lateralization of cognitive processes in the brain. Acta Psychol. 105, 211-235. doi: 10.1016/S0001-6918(00)00062-7

Itier, R. J., Latinus, M., and Taylor, M. J. (2006). Face, eye and object early processing: what is the face specificity? Neuroimage 29, 667-676. doi: 10.1016/j.neuroimage.2005.07.041

Itier, R. J., and Taylor, M. J. (2002). Inversion and contrast polarity reversal affect both encoding and recognition processes of unfamiliar faces: a repetition study using ERPs. Neuroimage 15, 353-372. doi: 10.1006/nimg.2001.0982

Itier, R. J., Van Roon, P., and Alain, C. (2011). Species sensitivity of early face and eye processing. Neuroimage 54, 705-713. doi: 10.1016/j.neuroimage.2010.07.031

Knudsen, E. I. (2007). Fundamental components of attention. Annu. Rev. Neurosci. 30, 57-78. doi: 10.1146/annurev.neuro.30.051606.094256

Leehey, S. C., and Cahn, A. (1979). Lateral asymmetries in the recognition of words, familiar faces and unfamiliar faces. Neuropsychologia 17, 619-628. doi: 10.1016/0028-3932(79)90036-8

Levine, S. C., Banich, M. T., and Koch-Weser, M. P. (1988). Face recognition: a general or specific right hemisphere capacity? Brain Cogn. 8, 303-325. doi: 10.1016/0278-2626(88)90057-7

Levine, S. C., and Koch-Weser, M. P. (1982). Right hemisphere superiority in the recognition of famous faces. Brain Cogn. 1, 10-22. doi: 10.1016/02782626(82)90003-3

Luck, S. (2005). An Introduction to the Event-Related Potential Technique. Cambridge: The MIT Press.

Luo, S., Luo, W., He, W., Chen, X., and Luo, Y. (2013). P1 and N170 components distinguish human-like and animal-like makeup stimuli. Neuroreport 24, 482486. doi: 10.1097/WNR.0b013e328361cf08

Martin, B. A., Tremblay, K. L., and Korczak, P. (2008). Speech evoked potentials: from the laboratory to the clinic. Ear. Hear. 29, 285-313. doi: 10.1097/AUD.0b013e3181662c0e

Maurer, U., Rossion, B., and McCandliss, B. D. (2008). Category specificity in early perception: face and word n170 responses differ in both lateralization and habituation properties. Front. Hum. Neurosci. 2:18. doi: 10.3389/neuro.09. 018.2008

Measso, G., and Zaidel, E. (1990). Effect of response programming on hemispheric differences in lexical decision. Neuropsychologia 28, 635-646. doi: 10.1016/00283932(90)90118-8

Mercure, E., Dick, F., Halit, H., Kaufman, J., and Johnson, M. H. (2008). Differential lateralization for words and faces: category or psychophysics? J. Cogn. Neurosci. 20, 2070-2087. doi: 10.1162/jocn.2008.20137 
Nieuwenhuis, S., Yeung, N., van den Wildenberg, W., and Ridderinkhof, K. R. (2003). Electrophysiological correlates of anterior cingulate function in a go/no-go task: effects of response conflict and trial type frequency. Cogn. Affect. Behav. Neurosci. 3, 17-26. doi: 10.3758/CABN.3.1.17

Ocklenburg, S., Güntürkün, O., and Beste, C. (2011). Lateralized neural mechanisms underlying the modulation of response inhibition processes. Neuroimage 55, 1771-1778. doi: 10.1016/j.neuroimage.2011.01.035

Ocklenburg, S., Güntürkün, O., and Beste, C. (2012). Hemispheric asymmetries and cognitive flexibility: an ERP and sLORETA study. Brain Cogn. 78, 148-155. doi 10.1016/j.bandc.2011.11.001

Ocklenburg, S., Ströckens, F., and Güntürkün, O. (2013). Lateralisation of conspecific vocalisation in non-human vertebrates. Laterality 18, 1-31. doi: 10.1080/1357650X.2011.626561

Oldfield, R. C. (1971). The assessment and analysis of handedness: the Edinburgh Inventory. Neuropsychologia 9, 97-113. doi: 10.1016/0028-3932(71)90067-4

Perrin, F., Pernier, J., Bertrand, O., and Echallier, J. F. (1989). Spherical splines for scalp potential and current density mapping. Electroencephalogr. Clin. Neurophysiol. 7, 184-187. doi: 10.1016/0013-4694(89) 90180-6

Proverbio, A. M., Mazzara, R., Riva, F., and Manfredi, M. (2012). Sex differences in callosal transfer and hemispheric specialization for face coding. Neuropsychologia 50, 2325-2332. doi: 10.1016/j.neuropsychologia.2012. 05.036

Proverbio, A. M., Riva, F., Martin, E., and Zani, A. (2010). Face coding is bilateral in the female brain. PLoS ONE 5:e11242. doi: 10.1371/journal.pone.0011242

Roche, R. A., Garavan, H., Foxe, J. J., and O'Mara, S. M. (2005). Individual differences discriminate event-related potentials but not performance during response inhibition. Exp. Brain Res. 160, 60-70. doi: 10.1007/s00221-0041985-z

Rossion, B., Campanella, S., Gomez, C. M., Delinte, A., Debatisse, D., Liard, L., et al. (1999). Task modulation of brain activity related to familiar and unfamiliar face processing: an ERP study. Clin. Neurophysiol. 110, 449-462. doi: 10.1016/S13882457(98)00037-6

Rossion, B., and Gauthier, I. (2002). How does the brain process upright and inverted faces? Behav. Cogn. Neurosci. Rev. 1, 63-75. doi: 10.1177/ 1534582302001001004

Rossion, B., Joyce, C. A., Cottrell, G. W., and Tarr, M. J. (2003). Early lateralization and orientation tuning for face, word, and object processing in the visual cortex. Neuroimage 20, 1609-1624. doi: 10.1016/j.neuroimage.2003.07.010

Sehlmeyer, C., Konrad, C., Zwitserlood, P., Arolt, V., Falkenstein, M., and Beste, C. (2010). ERP indices for response inhibtion are related to anxiety-related personality traits. Neuropsychologia 48, 2488-2495. doi: 10.1016/j.neuropsychologia.2010.04.022

Shibata, T., Nishijo, H., Tamura, R., Miyamoto, K., Eifuku, S., Endo, S., et al. (2002). Generators of visual evoked potentials for faces and eyes in the human brain as determined by dipole localization. Brain Topogr. 15, 51-63. doi: 10.1023/A:1019944607316

Smith, J. L., and Douglas, K. M. (2011). On the use of event-related potentials to auditory stimuli in the Go/NoGo task. Psychiatry Res. 193, 177-181. doi.org/10.1016/j.pscychresns.2011.03.002

Smith, J. L., Jamadar, S., Provost, A. L., and Michie, P. T. (2013). Motor and non-motor inhibition in the Go/NoGo task: an ERP and fMRI study. Int. J. Psychophysiol. 87, 244-253. doi: 10.1016/j.ijpsycho.2012. 07.185
Smith, J. L., Smith, E. A., Provost, A. L., and Heathcote, A. (2010). Sequence effects support the conflict theory of $\mathrm{N} 2$ and $\mathrm{P} 3$ in the Go/NoGo task. Int. J. Psychophysiol. 75, 217-226. doi: 10.1016/j.ijpsycho.2009.11.002

Sung, Y. W., Someya, Y., Eriko, Y., Choi, S. H., Cho, Z. H., and Ogawa, S. (2011). Involvement of low-level visual areas in hemispheric superiority for face processing. Brain Res. 1390, 118-125. doi: 10.1016/j.brainres.2011. 03.049

Schweinberger, S. R., Pickering, E. C., Jentzsch, I., Burton, A. M., and Kaufmann, J. M. (2002). Event-related brain potential evidence for a response of inferior temporal cortex to familiar face repetitions. Brain Res. Cogn. Brain Res. 14, 398409. doi: 10.1016/S0926-6410(02)00142-8

Taylor, M. J. (2002). Non-spatial attentional effects on P1. Clin. Neurophysiol. 113, 1903-1908. doi: 10.1016/S1388-2457(02)00309-7

Thoma, P., Soria Bauser, D., and Suchan, B. (2012). BESST (bochum emotional stimulus set) - a pilot validation study of a stimulus set containing emotional bodies and faces from frontal and averted view. Psychiatry Res. 209, 98-109. doi: 10.1016/j.psychres.2012.11.012

Volberg, G., and Hübner, R. (2004). On the role of response conflicts and stimulus position for hemispheric differences in global/local processing: an ERP study. Neuropsychologia 42, 1805-1813. doi: 10.1016/j.neuropsychologia.2004. 04.017

Wild-Wall, N., Falkenstein, M., and Gajewski, P. D. (2012). Neural correlates of changes in a visual search task due to cognitive training in seniors. Neural Plast. 2012, 529057. doi: 10.1155/2012/529057

Young, A. W. (1984). Right cerebral hemisphere superiority for recognizing the internal and external features of famous faces. Br. J. Psychol. 75, 161-169. doi: 10.1111/j.2044-8295.1984.tb01887.x

Young, A. W., and Bion, P. J. (1981). Accuracy of naming laterally presented known faces by children and adults. Cortex 17, 97-106. doi: 10.1016/S00109452(81)80009-3

Young, A. W., Hay, D. C., McWeeny, K. H., Ellis, A. W., and Barry, C. (1985). Familiarity decisions for faces presented to the left and right cerebral hemispheres. Brain Cogn. 4, 439-450. doi: 10.1016/0278-2626(85)90032-6

Yovel, G., Levy, J., and Yovel, I. (2001). Hemispheric asymmetries for global and local visual perception: effects of stimulus and task factors. J. Exp. Psychol. Hum. Percept. Perform. 27, 1369-1385. doi: 10.1037/0096-1523.27.6.1369

Conflict of Interest Statement: The authors declare that the research was conducted in the absence of any commercial or financial relationships that could be construed as a potential conflict of interest.

Received: 05 July 2013; accepted: 04 November 2013; published online: 22 November 2013.

Citation: Ocklenburg S, Ness V, Güntürkün O, Suchan B and Beste C (2013) Response inhibition is modulated by functional cerebral asymmetries for facial expression perception. Front. Psychol. 4:879. doi: 10.3389/fpsyg.2013.00879

This article was submitted to Cognition, a section of the journal Frontiers in Psychology. Copyright (c) 2013 Ocklenburg, Ness, Güntürkün, Suchan and Beste. This is an open-access article distributed under the terms of the Creative Commons Attribution License (CC BY). The use, distribution or reproduction in other forums is permitted, provided the original author(s) or licensor are credited and that the original publication in this journal is cited, in accordance with accepted academic practice. No use, distribution or reproduction is permitted which does not comply with these terms. 